\title{
Crystal orientation dependent intersubband transition in semipolar AIGaN/GaN single quantum well for optoelectronic applications
}

Houqiang Fu, Zhijian Lu, Xuanqi Huang, Hong Chen, and Yuji Zhao

Citation: Journal of Applied Physics 119, 174502 (2016); doi: 10.1063/1.4948667

View online: http://dx.doi.org/10.1063/1.4948667

View Table of Contents: http://aip.scitation.org/toc/jap/119/17

Published by the American Institute of Physics

\section{Articles you may be interested in}

Theoretical analysis of modulation doping effects on intersubband transition properties of semipolar AIGaN/GaN quantum well

Journal of Applied Physics 121, 014501 (2017); 10.1063/1.4972975

Analysis of low efficiency droop of semipolar InGaN quantum well light-emitting diodes by modified rate equation with weak phase-space filling effect

AIP Advances 6, 065013 (2016); 10.1063/1.4954296

Nonpolar and semipolar InGaN/GaN multiple-quantum-well solar cells with improved carrier collection efficiency Applied Physics Letters 110, 161105 (2017); 10.1063/1.4980139

Terahertz intersubband photodetectors based on semi-polar GaN/AIGaN heterostructures

Applied Physics Letters 108, 201102 (2016); 10.1063/1.4950852

Analysis of loss mechanisms in InGaN solar cells using a semi-analytical model

Journal of Applied Physics 119, 213101 (2016); 10.1063/1.4953006

Strain-induced polarization in wurtzite III-nitride semipolar layers

Journal of Applied Physics 100, 023522 (2006); 10.1063/1.2218385

\section{AlP | Journal of AlP Applied Physics}

Save your money for your research.

It's now FREE to publish with us no page, color or publication charges apply.

\section{Publish your research in the} Joumal of Applied Physics

to claim your place in applied

physics history. 


\title{
Crystal orientation dependent intersubband transition in semipolar AIGaN/GaN single quantum well for optoelectronic applications
}

\author{
Houqiang Fu, Zhijian Lu, Xuanqi Huang, Hong Chen, and Yuji Zhao \\ School of Electrical, Computer and Energy Engineering, Arizona State University, Tempe, \\ Arizona 85287, USA
}

(Received 23 February 2016; accepted 23 April 2016; published online 5 May 2016)

\begin{abstract}
The optical properties of intersubband transition in a semipolar $\mathrm{AlGaN} / \mathrm{GaN}$ single quantum well (SQW) are theoretically studied, and the results are compared with polar $c$-plane and nonpolar $m$-plane structures. The intersubband transition frequency, dipole matrix elements, and absorption spectra are calculated for SQW on different semipolar planes. It is found that SQW on a certain group of semipolar planes $\left(55^{\circ}<\theta<90^{\circ}\right.$ tilted from $c$-plane) exhibits low transition frequency and long wavelength response with high absorption quantum efficiency, which is attributed to the weak polarization-related effects. Furthermore, these semipolar SQWs show tunable transition frequency and absorption wavelength with different quantum well thicknesses, and stable device performance can be achieved with changing barrier thickness and $\mathrm{Al}$ compositions. All the results indicate that the semipolar $\mathrm{AlGaN} / \mathrm{GaN}$ quantum wells are promising candidate for the design and fabrication of high performance low frequency and long wavelength optoelectronic devices. Published by AIP Publishing. [http://dx.doi.org/10.1063/1.4948667]
\end{abstract}

\section{INTRODUCTION}

Wurtzite (Al, Ga, In)N wide bandgap III-nitride semiconductors have attracted tremendous attention due to their successful applications in blue and green light-emitting diodes (LEDs) and laser diodes (LDs), which enable efficient solid-state lighting and full-color displays. ${ }^{1-5}$ Recently, III-nitride materials have also been studied for intersubband transition (ISBT) based optoelectronics such as quantum well infrared photodetectors (QWIPs) ${ }^{6}$ quantum cascade detectors (QCDs), ${ }^{7}$ and quantum cascade lasers (QCLs). ${ }^{8}$ Because of their advantageous material properties such as large longitudinal photon energy and large band offset, ${ }^{8}$ they offer excellent device performance for high temperature low-frequency (such as 1-10 THz) and long-wavelength (such as infrared 30-300 $\mu \mathrm{m}$ ) applications. ${ }^{9,10}$

Because of different polarization-related effects, IIInitride materials can be divided into three categories: polar $c$-plane when $\theta=0^{\circ}$, semipolar planes when $0^{\circ}<\theta<90^{\circ}$, and nonpolar plane (typically $m$-plane) when $\theta=90^{\circ}$, where $\theta$ is the inclination angle of the crystal plane from $c$-plane. ${ }^{11}$ Till date, the majority of the studies on III-nitride ISBT has been focused on conventional polar $c$-plane III-nitride materials. ${ }^{6-10}$ However, these $c$-plane ISBT devices suffer from the titled quantum well (QW) profile due to the large polarization-induced electric field inside the QWs. As a result, most ISBT of these $c$-plane devices is limited to short wavelength regime. For example, lizuka et al. showed GaN/ AlN QWs with ISBT in the wavelength range of 1.3-2.2 $\mu \mathrm{m} .{ }^{12}$ Gmachl et al. reported ISBT of AlGaN/GaN multiple QWs at wavelengths of $1.75-4.2 \mu \mathrm{m}$ grown on $c$-plane sapphire substrate. ${ }^{13}$ Though few reports demonstrated THz transition on the $c$-plane GaN QW, it involved complicated structure design and was operated at an extremely low temperature of $4 \mathrm{~K} .{ }^{14} \mathrm{In}$ order to get rid of the polarization-related effects, the ISBT device structures based on novel nonpolar $m$-plane (with crystal inclination angle $\theta=90^{\circ}$ from $c$-plane) III-nitride materials have been proposed. Feezell et al. theoretically studied the ISBT properties of nonpolar III-nitride QWs and found that their absorption wavelengths are longer than those of the $c$-plane QWs. ${ }^{15}$ Pesach et al. fabricated low temperature $(14 \mathrm{~K})$ nonpolar InGaN/AlGaN QW infrared photodetectors with a longest wavelength of $9.3 \mu \mathrm{m} .{ }^{16}$ Edmunds $e t a l$. reported THz ISBT in the $m$-plane AlGaN/GaN QWs. ${ }^{17}$ However, the operation temperature $(9 \mathrm{~K})$ was too low for practical applications. Other reports can be found in Ref. 18. Despite their appealing properties, the growth challenges such as stacking faults and alloy inhomogeneity ${ }^{19-21}$ have make it very difficult to fabricate high quality nonpolar QWs. Recently, optoelectronic devices grown on semipolar III-nitride substrates $\left(0^{\circ}<\theta<90^{\circ}\right)$ have gained considerable interests, and high performance LEDs and LDs have been achieved. ${ }^{21-25}$ The advantage of semipolar QWs over nonpolar structures is the much improved material epitaxial quality ${ }^{21}$ while keeping the weak polarization properties. ${ }^{11}$ Researchers from CNRS group first studied the ISBT on $(11 \overline{2} 2)$ GaN/AIN QWs with wavelength of $1.5-4.5 \mu \mathrm{m},{ }^{26,27}$ which is comparable to some $m$-plane devices. ${ }^{18}$ Despite this encouraging result, the report on semipolar III-nitride ISBT is very limited, and their fundamental physical process is still unclear.

In this work, we systematically study theoretical optical properties of ISBT for AlGaN/GaN single quantum well (SQW) on semipolar III-nitride planes, while results on polar $c$-plane and nonpolar $m$-plane are also obtained. By examining the ISBT frequency, dipole matrix elements, and absorption spectra, we find that nonpolar and certain semipolar planes $\left(55^{\circ}<\theta<90^{\circ}\right)$ have the optimal performance for ISBT devices with high absorption quantum efficiency (QE). SQW on these semipolar planes show low transition energy 
and long wavelength response by increasing QW thickness. The paper is organized as follows: in Section II, we describe the details of the simulation methods; in Section III A, we study ISBT properties of different semipolar AlGaN/GaN SQW and identify suitable semipolar polar orientations for device applications; in Section III B, two specific semipolar planes $(10 \overline{1} 3)\left(\theta=32^{\circ}\right.$, strong polarization) and $(20 \overline{2} 1)$ $\left(\theta=75^{\circ}\right.$, weak polarization) are studied with different $\mathrm{QW}$ thicknesses, barrier thicknesses, and barrier Al composition, and their device performances are compared.

\section{SIMULATION METHODS}

ISBT properties of semipolar AlGaN/GaN SQW are theoretically studied for optoelectronic applications, or specifically for photodetectors. QW structures with different QW thicknesses, barrier thicknesses, and barrier Al composition are calculated and the results are discussed. To calculate the QW bandstructures and subband wavefunctions, we use a commercial software SiLENSe developed by STR Group, ${ }^{28}$ where one-dimensional Schrödinger-Poisson equation is solved self-consistently with drift-diffusion model included based on the effective mass approximation. The simulation includes the effects of strain and polarization for arbitrary crystal orientations (i.e., polar, nonpolar, and semipolar) of III-nitride materials, which are critical for this work. The elastic constants and piezoelectric coefficients of the software used are listed in Table I. For AlGaN/GaN heterostructure, the total polarization $\left(\mathrm{P}_{\text {tot }}\right)$ is the sum of piezoelectric polarization $\mathrm{P}_{\mathrm{pz}}$ and spontaneous polarization $\mathrm{P}_{\mathrm{sp}}$. The polarization charge $\sigma$ at the interface is given by ${ }^{29}$

$$
\begin{aligned}
\sigma & =\left(P_{p z}^{A l G a N}+P_{s p}^{A l G a N}\right)-\left(P_{p z}^{G a N}+P_{s p}^{G a N}\right) \\
& =-\left(\Delta P_{p z}+\Delta P_{s p}\right)
\end{aligned}
$$

where $P_{p z}^{A l G a N}$ and $P_{s p}^{A l G a N}$ are the piezoelectric and spontaneous polarization of $\mathrm{AlGaN}$ barrier, respectively; $P_{p z}^{G a N}$ and $P_{s p}^{G a N}$ are the piezoelectric and spontaneous polarization of the GaN QW layer, respectively; and $\Delta \mathrm{P}_{\mathrm{pz}}$ and $\Delta \mathrm{P}_{\mathrm{sp}}$ are the difference in piezoelectric and spontaneous polarization between GaN QW layer and AlGaN barrier layer. For a nonpolar or semipolar plane titled from $c$-plane by an angle of $\theta$, the total polarization change that determines the interface charge is expressed as a function of $\theta$ by

$$
\Delta P_{t o t}=P_{p z}^{G a N}+\left(P_{s p}^{G a N}-P_{s p}^{A l G a N}\right) \cos \theta,
$$

where $\Delta \mathrm{P}_{\text {tot }}$ is the total difference of polarization between the GaN layer. $P_{p z}^{G a N}$ is the strain-induced piezoelectric

\begin{tabular}{|c|c|c|c|c|c|c|c|c|c|}
\hline & \multicolumn{5}{|c|}{ Elastic constant $(\mathrm{GPa})$} & \multicolumn{4}{|c|}{ Piezoelectric coefficient $\left(\mathrm{C} / \mathrm{cm}^{2}\right)$} \\
\hline & $\mathrm{C}_{11}$ & $\mathrm{C}_{12}$ & $\mathrm{C}_{13}$ & $\mathrm{C}_{33}$ & $\mathrm{C}_{44}$ & $\mathrm{e}_{15}$ & $\mathrm{e}_{31}$ & $\mathrm{e}_{33}$ & $\mathrm{P}_{\mathrm{sp}}$ \\
\hline $\mathrm{GaN}$ & 375 & 140 & 105 & 395 & 100 & -0.27 & -0.33 & 0.65 & -0.029 \\
\hline $\mathrm{AlN}$ & 395 & 140 & 115 & 385 & 120 & -0.48 & -0.58 & 1.55 & -0.081 \\
\hline
\end{tabular}

TABLE I. Elastic constants and piezoelectric coefficients used in SiLENSe to calculate spontaneous polarization and piezoelectric polarization. polarization in the GaN layer on top of the AlGaN barrier layer, which can be expressed as

$$
\begin{aligned}
P_{p z}^{G a N}= & e_{31} \cos \theta \epsilon_{x^{\prime} x^{\prime}}+\left(e_{31} \cos ^{3} \theta+\frac{e_{33}-e_{15}}{2} \sin \theta \sin 2 \theta\right) \epsilon_{y^{\prime} y^{\prime}} \\
& +\left(\frac{\left(e_{31}+e_{15}\right)}{2} \sin \theta \sin 2 \theta+e_{33} \cos ^{3} \theta\right) \epsilon_{z^{\prime} z^{\prime}} \\
& +\left[\left(e_{31}-e_{33}\right) \cos \theta \sin 2 \theta+e_{15} \sin \theta \cos 2 \theta\right] \epsilon_{y^{\prime} z^{\prime}}
\end{aligned}
$$

where elements $\varepsilon_{\mathrm{k}^{\prime} \mathrm{m}^{\prime}}$ are the strain tensor components and elements $\mathrm{e}_{\mathrm{ij}}$ are the components of piezoelectric tensor in Voigt notation. From Eqs. (1)-(3), we can see that the polarization and the interface charge are highly dependent on the crystal orientation, which could significantly modify device performances. More detailed calculations of strain and piezoelectric charge in nonpolar and semipolar SQW can be found in Ref. 11. After including the polarization effect, the band diagram, subband wavefunction, and carrier distribution can be calculated using the material parameters listed in Table II. The material properties of alloys are obtained based on the Vegard's law. More information about SiLENSe and publications using this software can be found in Ref. 30 .

In a QW system, the electron concentration $n_{i}$ of the $i$ th subband is calculated by the integral of Fermi-Dirac distribution and density of states as follows:

$$
n_{i}=\int_{E_{i}}^{\infty} \frac{m^{*}}{\pi \hbar^{2}} \frac{1}{1+e^{\frac{E-E_{f}}{k T}}} d E=\frac{m^{*} k T}{\pi \hbar^{2}} \ln \left(1+e^{\frac{E_{f}-E_{i}}{k T}}\right),
$$

where $\mathrm{m}^{*}$ is the effective mass, $k$ is the Boltzmann constant, $\mathrm{T}$ is the operation temperature, $h$ is the Planck's constant, $\mathrm{E}_{\mathrm{f}}$ is the Fermi energy, and $E_{\mathrm{i}}$ is the energy of $i$ th subband. Incorporating the difference in electron concentration between the first two subbands, the ISBT absorption coefficient $\alpha(\lambda)$ between the first two subbands is derived using the Fermi golden rule and can be expressed as follows: $:^{31,32}$

$$
\begin{aligned}
\alpha(\lambda)= & \frac{8 \pi^{3} c^{2} \mu}{\lambda n_{r} L} \sin ^{2} \gamma\left|M_{12}\right|^{2} \frac{m^{*} k T}{\pi h^{2}} \ln \left\{\frac{1+\exp \left[\left(E_{f}-E_{1}\right) / k T\right]}{1+\exp \left[\left(E_{f}-E_{2}\right) / k T\right]}\right\} \\
& \times \frac{h /(2 \pi \tau)}{\left(E_{2}-E_{1}-h c / \lambda\right)^{2}+[h /(2 \pi \tau)]^{2}}
\end{aligned}
$$

TABLE II. Material parameters used in SiLENSe software for calculating conduction band, electron wavefunction, and electron concentration.

\begin{tabular}{lccc}
\hline \hline Material parameter & Unit & AlN & GaN \\
\hline Energy gap & $\mathrm{eV}$ & 6.25 & 3.51 \\
Varshni parameter a & $\mathrm{meV} / \mathrm{K}$ & 1.799 & 0.909 \\
Varshni parameter b & $\mathrm{K}$ & 1462 & 830 \\
Electron affinity & $\mathrm{eV}$ & 0 & 1.96 \\
Dielectric constant & $\ldots$ & 8.5 & 8.9 \\
Donor ionization energy & $\mathrm{meV}$ & 13 & 13 \\
Electron $g$-factor & $\ldots$ & 2 & 2 \\
Electron effective mass along axis $a$ & $\mathrm{~m}_{0}$ & 0.26 & 0.2 \\
Electron effective mass along axis $c$ & $\mathrm{~m}_{0}$ & 0.25 & 0.26 \\
Lattice constant $a$ & $\mathrm{~nm}$ & 0.3112 & 0.3188 \\
Lattice constant $c$ & $\mathrm{~nm}$ & 0.4982 & 0.5186 \\
\hline \hline
\end{tabular}


where $c$ is the speed of light in vacuum, $\mu$ is the permeability of QW material ( $\mathrm{GaN}$ in this case), $\lambda$ is wavelength of incident light, $\mathrm{n}_{\mathrm{r}}$ is refractive index, $\mathrm{L}$ is the QW thickness, $\gamma$ is the light propagation angle (usually $45^{\circ}$ is used to meet the polarization selection rule and increase the light absorption length $\left.{ }^{32}\right), M_{12}$ is the dipole matrix element of first two subbands, and $\tau$ is the relaxation time. In the calculation of absorption coefficient, the AlGaN barrier is unintentionally doped $\left(10^{15} \mathrm{~cm}^{-3}\right)$, and the QW is doped in a way to maximize the background limited infrared performance (BLIP) temperature. ${ }^{15}$ In the simulation of the conduction bands in Figs. 1 and 9, the QW is undoped for clear manifestation of the polarization effect on different planes, since high QW doping will cause band bending and make it difficult to compare. We will investigate in detail the doping effect in the future publications. Here, we mainly focus on the polarization effect on the transition energy and absorption coefficient and thus do not include the many-body effects. In the calculation of absorption coefficient, we use single effective mass of 0.2 $\mathrm{m}_{0}$, where $\mathrm{m}_{0}$ is free electron mass. From Table II, the electron effective mass along $a$ and $c$ axes are all $0.2 \mathrm{~m}_{0}$ for GaN. Though the effective mass is dependent on the crystal orientation, this dependence is noticeable only on hole not on the electron effective mass. ${ }^{33}$ The variation in electron effective mass is relatively small, and the impact on ISBT properties is neglected for simplicity. In this work, the temperature $\mathrm{T}$ is set as $300 \mathrm{~K} . \mathrm{n}_{\mathrm{r}}$ for $\mathrm{GaN}$ is $2.30 .^{34} \mu$ is calculated by $\mathrm{n}_{\mathrm{r}}^{2} \mu_{0} / \varepsilon_{\mathrm{r}}$, where $\mu_{0}$ is the vacuum permeability and $\varepsilon_{\mathrm{r}}$ is the relative dielectric constant for GaN (5.37 is used ${ }^{35}$ ). The relaxation time $\tau$ is determined by the Lorentzian linewidth of the absorption spectrum and $66 \mathrm{fs}$ is used in this simulation. ${ }^{15}$

The dipole matrix element $\mathrm{M}_{12}$ is defined as

$$
M_{12}=e \int_{-\infty}^{\infty} \psi_{2}^{*}(z) z \psi_{1}(z) d z
$$

where $e$ is the electron charge, $z$ is the along the device growth direction, $\psi_{1}$ is wavefuction of the first subband, and $\psi_{2}$ is the wavefunction of the second subband.

\section{RESULTS AND DISSCUSSION}

In Section III A, we first discuss the ISBT properties of AlGaN/GaN SQW on different crystal orientations. We observed unique properties for certain semipolar planes $\left(55^{\circ}<\theta<90^{\circ}\right)$ : low ISBT frequency, long wavelength response, and high absorption, which are promising for ISBT optoelectronics. In Section III B, we perform detailed studies on QW structures on two particular semipolar planes with different polarization effects, i.e., $(10 \overline{1} 3)\left(\theta=32^{\circ}\right.$, strong polarization) and (20121) $\left(\theta=75^{\circ}\right.$, weak polarization), where the polar $c$-plane and the nonpolar $m$-plane SQWs are also calculated as references. In Section III B 1, we focused on the QW thickness of the structures, which is varied from $3 \mathrm{~nm}$ to $12 \mathrm{~nm}$. The semipolar (202) 1$) \mathrm{SQW}$ shows tunable ISBT frequency down to sub-10 THz and absorption wavelength up to $30 \mu \mathrm{m}$. In Sections III B 2 and III B 3, we investigate the ISBT properties of SQW with various barrier thicknesses and Al composition. The polar c-plane and (1013) show higher transition frequency, shorter absorption wavelength, and smaller absorption with increasing barrier thickness and $\mathrm{Al}$ composition, which degrades device performance. In contrast, semipolar (202̄1) SQW shows stable ISBT properties.

\section{A. Crystal orientation}

In this section, we investigate the effect of crystal orientation $\theta$ on the ISBT properties of $\mathrm{Al}_{0.3} \mathrm{Ga}_{0.7} \mathrm{~N}(25 \mathrm{~nm}) / \mathrm{GaN}$ $(5 \mathrm{~nm}) \mathrm{SQW}$ structures on top of $200 \mathrm{~nm} \mathrm{n-GaN.} \mathrm{30 \%} \mathrm{of} \mathrm{alu-}$ minum is selected based on the common design of GaAsbased QWIPs. In the polar $c$-plane SQW, spontaneous
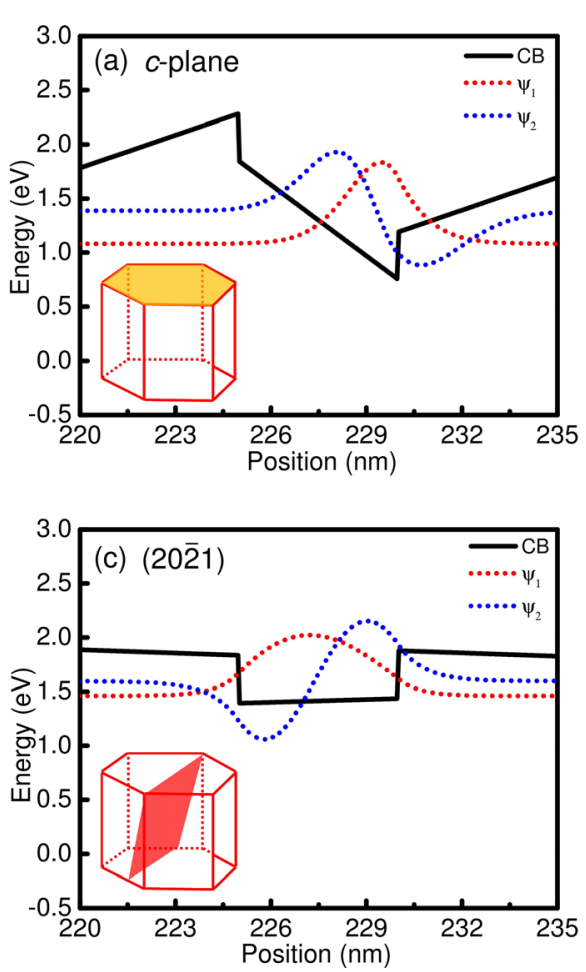
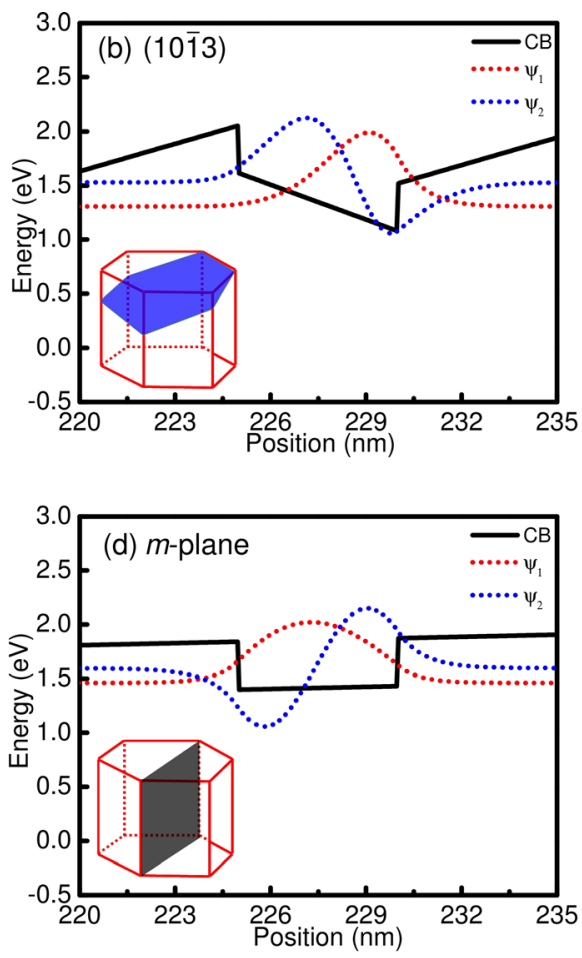

FIG. 1. Conduction band (CB) of $\mathrm{Al}_{0.3} \mathrm{Ga}_{0.7} \mathrm{~N} \quad(25 \mathrm{~nm}) / \mathrm{GaN} \quad(5 \mathrm{~nm})$ undoped SQW on (a) $c$-plane, (b)

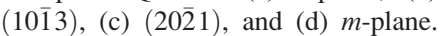
The first two subbands' wavefunction $\psi_{1}$ and $\psi_{2}$ are also shown. 
polarization and strain induced piezoelectric polarization result in strong electric field inside the QW. In the nonpolar $m$-plane case, the polarization is normal to growth direction, which means the polarization-related electric fields do not affect the QW profile. Between these two planes, semipolar planes have reduced polarization effect. In Fig. 1, we calculate the undoped QW conduction band profile of $c$-plane,

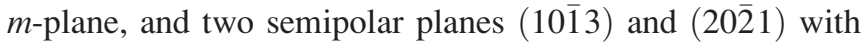
the same structure under zero bias. The polar $c$-plane $\left(\theta=0^{\circ}\right)$ and semipolar $(10 \overline{1} 3)\left(\theta=32^{\circ}\right)$ SQW show tilted bandstructure while the $m$-plane $\left(\theta=90^{\circ}\right)$ and semipolar $(20 \overline{2} 1)\left(\theta=75^{\circ}\right)$ have relatively flat profile. Due to the titled band profile, the first two subband wavefunction $\psi_{1}$ and $\psi_{2}$ from $c$-plane and semipolar (1013) structures are largely separated in terms of both energy and position, which will influence dipole matrix elements, ISBT frequency, and absorption coefficients. These results clearly show that polarization-related effects have significant impacts on the ISBT performance of AlGaN/GaN SQW.

Figure 2 shows ISBT frequencies and matrix elements and for crystal orientation $\theta$ from $0^{\circ}$ to $90^{\circ}$, where a wide range of semipolar structures are covered. The transition frequency is given by $\left(\mathrm{E}_{2}-\mathrm{E}_{1}\right) / h$. It is noteworthy that the "pair" semipolar planes with different polarities [e.g., (20다) at $75^{\circ}$ and $(20 \overline{2} \overline{1})$ at $105^{\circ}$ ] have almost identical in their ISBT properties (calculation not shown here). Although the Ga-polar and N-polar QW profiles have opposite QW tilting directions, the impacts on the dipole matrix elements and energy difference of first two subbands are minimum. Therefore, the semipolar structures with $\theta$ from $90^{\circ}$ to $180^{\circ}$ will have similar results to this work. In Fig. 2, the ISBT frequency (in blue) decreases monotonically with $\theta$ varying from $0^{\circ}$ to $55^{\circ}$. When $\theta$ is larger than $55^{\circ}$, minimum difference of ISBT frequency is observed. This trend of transition frequency can be directly linked to the polarization-related effects. With weaker polarizations, QW profile is less titled and the wavefunctions of subbands are less separated, which will lead to a smaller transition frequency. When a semipolar plane is $45^{\circ}$ titled from $c$-plane, $\Delta \mathrm{P}_{\mathrm{pz}}=0$, which is marked

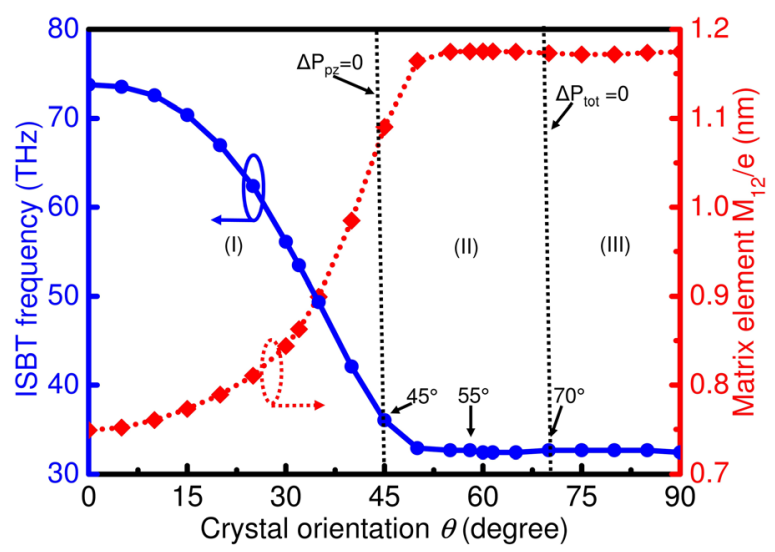

FIG. 2. ISBT frequency and matrix element $\mathrm{M}_{12} / \mathrm{e}$ and of $\mathrm{Al}_{0.3} \mathrm{Ga}_{0.7} \mathrm{~N}$ $(25 \mathrm{~nm}) / \mathrm{GaN}(5 \mathrm{~nm}) \mathrm{SQW}$ structures on various semipolar plane orientations. The first dashed line is at $\theta=45^{\circ}$ where piezoelectric polarization $\Delta \mathrm{P}_{\mathrm{pz}}=0$; the second dashed line is at $\theta=70^{\circ}$ where the total polarization $\Delta \mathrm{P}_{\text {tot }}=0$. Three regions (I), (II), and (III) are defined by the two dashed lines. by the first dashed line in Fig. 2. Although the reduced $\Delta \mathrm{P}_{\mathrm{pz}}$ leads to the decreasing of transition frequency from $0^{\circ}$ to $45^{\circ}$, the addition of $\Delta \mathrm{P}_{\mathrm{sp}}$ moves the crossover of $\Delta \mathrm{P}_{\text {tot }}$ to $\theta=70^{\circ}$, which is marked by the second dashed line in Fig. 2. According to the results in Fig. 2, all the semipolar planes can be divided into three regions: in region $\mathrm{I}\left(0^{\circ}<\theta<45^{\circ}\right)$, the $\Delta \mathrm{P}_{\mathrm{pz}}$ is the dominant effect; in region II $\left(45^{\circ}<\theta<70^{\circ}\right)$, both $\Delta \mathrm{P}_{\mathrm{pz}}$ and $\Delta \mathrm{P}_{\mathrm{sp}}$ are playing significant roles; in region III $\left(70^{\circ}<\theta<90^{\circ}\right), \Delta \mathrm{P}_{\text {tot }} \approx 0$. According to Fig. 2, although the crossover of $\Delta \mathrm{P}_{\text {tot }}$ happens at $\theta=70^{\circ}, \Delta \mathrm{P}_{\text {tot }}$ is already small enough when $\theta>55^{\circ}$ and has negligible effects on the $\mathrm{QW}$ profile. The simulation shows that the transition frequency of the semipolar $(20 \overline{2} 1)$ structure (region III) can go down to $30 \mathrm{THz}$, while the frequency of the $c$-plane structure is still over $80 \mathrm{THz}$. Therefore, the nonpolar and semipolar planes with $\theta>55^{\circ}$ are preferable for lower frequency application. In addition, an opposite trend was also observed for the dipole matrix elements (in red), which can also be explained by the polarization effects. It is shown that semipolar structures with $\theta>55^{\circ}$ have very large matrix elements and possibly high absorption coefficients, which is another advantageous feature.

In Fig. 3(a), we calculate the absorption spectra for SQW on the polar $c$-plane, nonpolar $m$-plane, and semipolar planes from three regions in Fig. 2. With increasing $\theta$, the spectra of the semipolar planes are moving towards those of the nonpolar $m$-plane. And the spectrum of (20) 1$)$ and $m$-plane almost overlap, which makes the semipolar $(20 \overline{2} 1)$ a good alternative of the nonpolar $m$-plane. Fig. 3(b) shows the peak absorption coefficient and the peak absorption wavelength of SQW with different $\theta$. When $0^{\circ}<\theta<55^{\circ}$, the peak absorption wavelength increases with increasing $\theta$. When $\theta>55^{\circ}$, both the parameters reached maxima and the wavelength is kept at $9 \mu \mathrm{m}$ for this particular structure. In terms of peak absorption coefficients, two plateaus are observed. The semipolar planes from regions II $\left(55^{\circ}<\theta<70^{\circ}\right)$ and III have the highest absorption compared with other semipolar plane orientations. The absorption quantum efficiency (QE) is a critical parameter especially for real device applications, which is given by $\alpha(\lambda) \mathrm{L} / \cos \gamma$, where $\mathrm{L} / \cos \gamma$ is the light propagation length in devices. ${ }^{32}$

The peak absorption QE as a function of peak absorption wavelength (blue squares) for various semipolar structures is illustrated in Fig. 4. Again, all the planes can be categorized into the same three regions: region I represents low absorption and short wavelength region; region II is the transition region; and region III is high absorption and long wavelength region. The results show that the nonpolar and semipolar planes in regions II (when $\theta>55^{\circ}$ ) and III can access to longer wavelength and have higher peak absorption QE, which are desirable for devices operating at long wavelength and low frequency regime. Furthermore, from the perspective of device growth, it is preferable to grow less QWs in order to ensure good material qualities, as the lattice mismatch significantly increases with the number of QWs. In Fig. 4, the $50 \%$ absorption QW numbers as a function of peak absorption wavelength (red circles) for different nonpolar and semipolar structures is also illustrated. The 50\% absorption QW numbers is the QW numbers when $50 \%$ of incident light are 


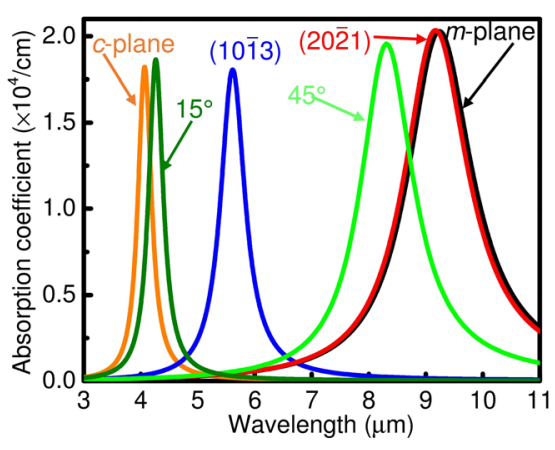

(a)

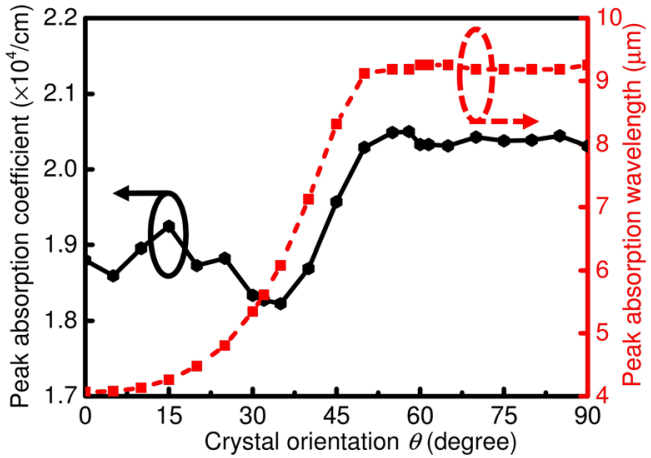

(b)
FIG. 3. (a) Absorption spectra for AlGaN/GaN SQW on different planes including $c$-plane, $m$-plane, and four semipolar planes. (b) Peak absorption coefficient and peak absorption wavelength as a function of semipolar plane orientation. absorbed per round-trip and is given by $\mathrm{N}=-\ln (50 \%) /($ peak absorption QE). ${ }^{13}$ It is shown that QW structures on the nonpolar and semipolar planes in regions I and II $\left(0^{\circ}<\theta<55^{\circ}\right)$ require more QWs to achieve $50 \%$ absorption than those on crystal planes in regions II $\left(55^{\circ}<\theta<70^{\circ}\right)$ and III. In short summary, the results from above two metrics indicate that QWs on the nonpolar and semipolar planes $\left(55^{\circ}<\theta<90^{\circ}\right)$ will facilitate better performance at longer wavelength regime and has lower material requirement, which are advantageous for device applications.

\section{B. Case studies: $c$-plane, $(\mathbf{1 0} \overline{13}),(20 \overline{1} 1)$, and $m$-plane}

In the following Sections III B 1-III B 3, we focus on two distinct semipolar planes $(10 \overline{1} 3)\left(\theta=32^{\circ}\right.$, strong polarization) and $(20 \overline{2} 1)\left(\theta=75^{\circ}\right.$, weak polarization) for more detailed studies, where results on the polar $c$-plane and nonpolar $m$-plane are also included as references. The QW thickness, barrier thickness, and barrier $\mathrm{Al}$ composition will be modified, and their effects on the ISBT properties will be discussed.

\section{Quantum well thickness}

The simulated SQW structure has a $25 \mathrm{~nm} \mathrm{Al}_{0.3} \mathrm{Ga}_{0.7} \mathrm{~N}$ barrier, where the thicknesses of QW is varied from $3 \mathrm{~nm}$ (typical thickness of $c$-plane $\mathrm{QW}$ ) to $12 \mathrm{~nm}$ (thickest $\mathrm{QW}$ reported for the semipolar planes ${ }^{36}$ ). Figure 5 shows the dipole matrix element and ISBT frequency of the SQW on the semipolar (20121), (1013), c-plane, and $m$-plane. The semipolar (2021) and nonpolar $m$-plane SQW have lower

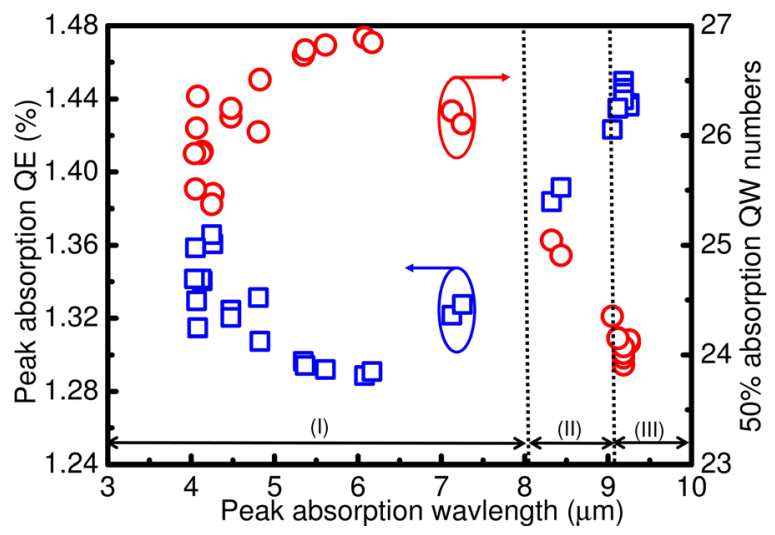

FIG. 4. Peak absorption QE and 50\% absorption QW numbers versus peak absorption wavelength, which has been divided into three regions of semipolar plane orientation based QW performance. transition frequency at any given QW thickness than that on the semipolar (1013) and c-plane SQW. Furthermore, the transition frequency of semipolar $(20 \overline{2} 1)$ plane decreases with increasing QW thickness. Due to largely reduced polarization-related effects, the QW profile on the semipolar $(20 \overline{2} 1)$ plane can be approximately treated as a square finite potential well, for which the quantum mechanical calculation indicates the energy separation of the subbands and transition frequency are inversely proportional to the well thickness. ${ }^{32}$ In contrast, for the semipolar (1013) structures with strong polarization-related effects, the transition frequency first decreases with increasing QW thickness and then stays almost constant. This is because of the triangular potential well on the semipolar $(10 \overline{1} 3)$ plane due to the large residual polarizations, which limits the separation of subband wavefunctions. This is more evident in the case of $c$-plane QW structures with the strongest polarization effects, where changing the QW thickness barely influences the transition frequency. In summary, the semipolar (2021) SQW shows great advantages with tunable ISB transition frequency reaching to a much lower frequency regime. In addition, both semipolar $(20 \overline{2} 1)$ and $m$-plane SQW have larger dipole matrix elements which contribute to higher absorption at the same QW thickness.

Figures 6(a)-6(c) show the absorption spectra for

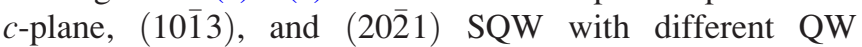
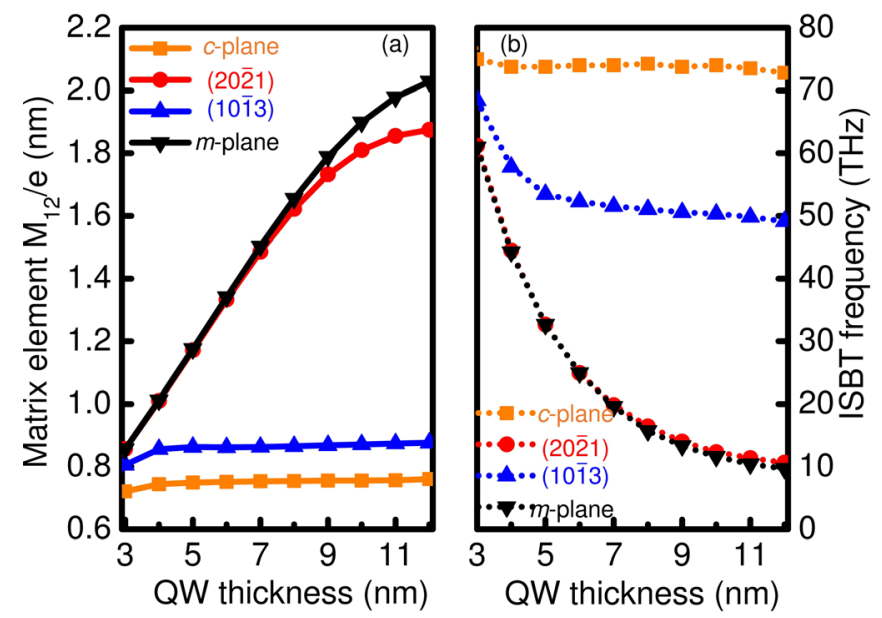

FIG. 5. (a) Matrix element $\mathrm{M}_{12} / e$ and (b) ISBT frequency of $\mathrm{Al}_{0.3} \mathrm{Ga}_{0.7} \mathrm{~N}$ $(25 \mathrm{~nm}) / \mathrm{GaN}$ SQW as a function of GaN QW thickness on $c$-plane, $(20 \overline{2} 1)$, (1013), and $m$-plane. 

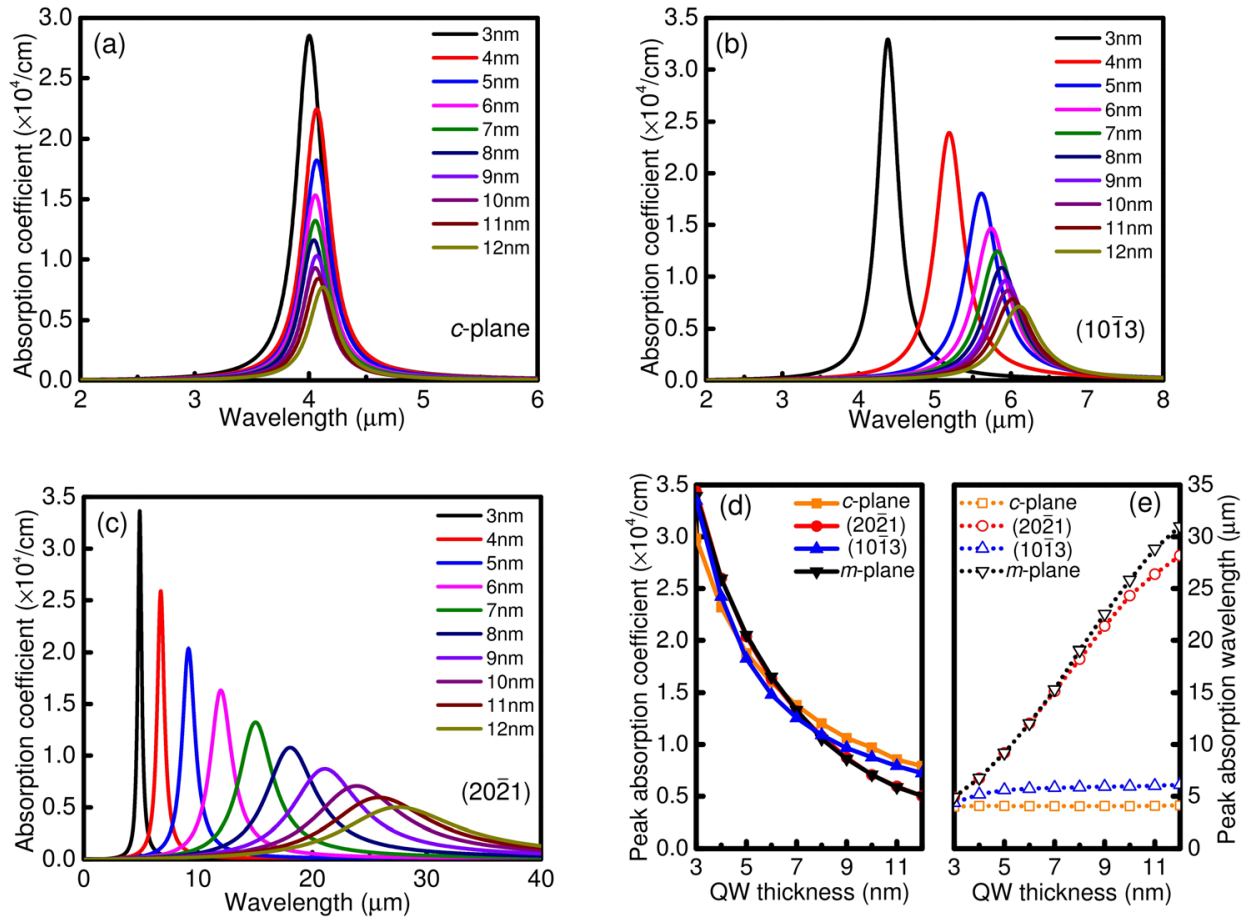

FIG. 6. Absorption spectra of (a)

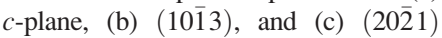
$25 \mathrm{~nm} \mathrm{Al}{ }_{0.3} \mathrm{Ga}_{0.7} \mathrm{~N} / \mathrm{GaN}$ SQW varying QW thickness from $3 \mathrm{~nm}$ to $12 \mathrm{~nm}$. Peak absorption coefficient (d) and peak absorption wavelength (e) as a function of QW thickness for c-plane, $(20 \overline{2} 1)$, (1013), and $m$-plane $\mathrm{Al}_{0.3} \mathrm{Ga}_{0.7} \mathrm{~N}$ $(25 \mathrm{~nm}) / \mathrm{GaN} \mathrm{SQW}$. thicknesses. Fig. 6(d) summarizes the peak absorption coefficient and peak absorption wavelength for different planes. All the spectra of the $c$-plane SQW are centralized at a peak wavelength around $4 \mu \mathrm{m}$. In the case of semipolar (1013) SQW, although the first two spectra are separated, the rest are accumulated at a peak wavelength of $6 \mu \mathrm{m}$. In contrast, the semipolar $(20 \overline{2} 1)$ SQW has much dispersive distribution of absorption spectra along the wavelength range. At QW thickness of $12 \mathrm{~nm}$, the peak absorption wavelength is around $28 \mu \mathrm{m}$ (far infrared). Therefore, the semipolar (20ㄹ) SQW can facilitate longer wavelength operation without a much reduction in absorption, which cannot be achieved using the semipolar (1013) plane. Peak absorption occurs when $h \mathrm{c} / \lambda=\mathrm{E}_{2}-\mathrm{E}_{1}$. For all four samples, the peak absorption coefficient decreases with increasing QW thickness. Though thicker QW will reduce the energy separation between the first two subbands, the electron occupation term in Eq. (5), which is proportional to $\left(n_{1}-n_{2}\right)$ according to Eq. (4), is less effected since $n_{1}$ is still much larger than $n_{2}$ and BILP condition is applied $\left(\mathrm{E}_{\mathrm{f}}-\mathrm{E}_{1}=\mathrm{kT}\right)$. The combination of increasing peak absorption wavelength and QW thickness reduces the peak absorption coefficients despite there is an increase in matrix element according to Eq. (5).

\section{Barrier thickness}

In this section, we study the ISBT properties of $\mathrm{Al}_{0.3} \mathrm{Ga}_{0.7} \mathrm{~N} / \mathrm{GaN}$ (5 nm) SQW with barrier thickness varying from $5 \mathrm{~nm}$ to $25 \mathrm{~nm}$. Figure 7 presents the dipole matrix elements and ISB transition frequency of semipolar (20 $\overline{2} 1)$, (1013), polar $c$-plane and nonpolar $m$-plane SQW. For the semipolar (1013) and $c$-plane SQW, the transition frequency dramatically increases with the barrier thickness and the dipole matrix elements are largely reduced. On the other hand, the semipolar $(20 \overline{2} 1)$ SQW does not show any degradation in the transition frequency and dipole matrix elements. Therefore, the semipolar $(20 \overline{2} 1)$ based ISBT devices could offer stable performance even though the barrier thickness are varied during device growth. Figures 8(a)-8(c) show the absorption spectra of SQW with various barrier thicknesses on different planes. Fig. 8(d) shows the peak absorption coefficient and peak absorption wavelength. In the case of the semipolar $(20 \overline{2} 1)$ SQW, almost all the spectra are overlapped at a peak absorption wavelength of $9 \mu \mathrm{m}$. For the semipolar (1013) and $c$-plane SQW, increasing barrier thickness moves their absorption spectra towards much shorter wavelength. Furthermore, the reduction in peak absorption coefficients is also observed for semipolar (1013) and $c$-plane SQW. Therefore, thick barriers will adversely affect the device performance of the semipolar (1013) and $c$-plane SQW, while have minimum influence on the semipo$\operatorname{lar}(20 \overline{2} 1)$ and $m$-plane SQW.
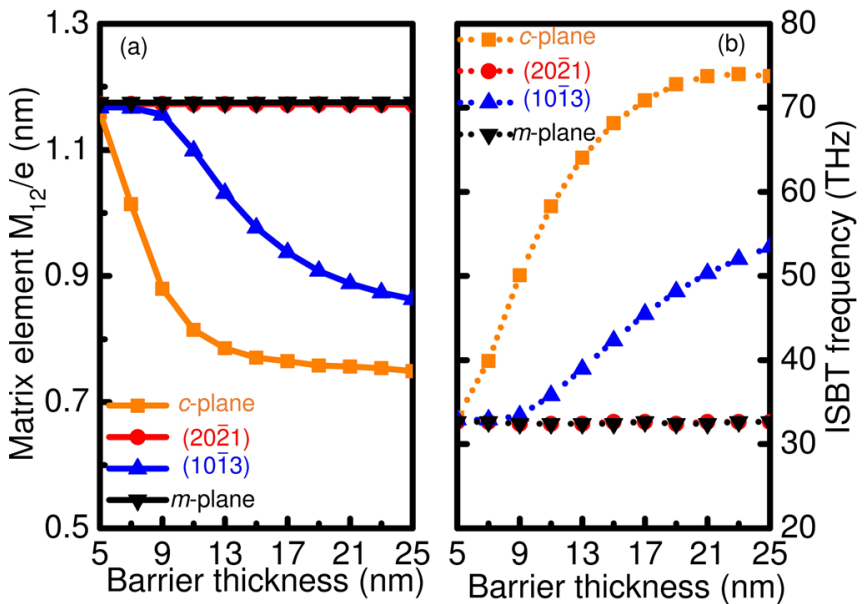

FIG. 7. (a) Matrix element $\mathrm{M}_{12} / e$ and (b) ISBT frequency of $\mathrm{Al}_{0.3} \mathrm{Ga}_{0.7} \mathrm{~N} /$ $\mathrm{GaN}(5 \mathrm{~nm}) \mathrm{SQW}$ as a function of barrier thickness on $c$-plane, $(20 \overline{\overline{2}} 1)$, (1013), and $m$-plane. 

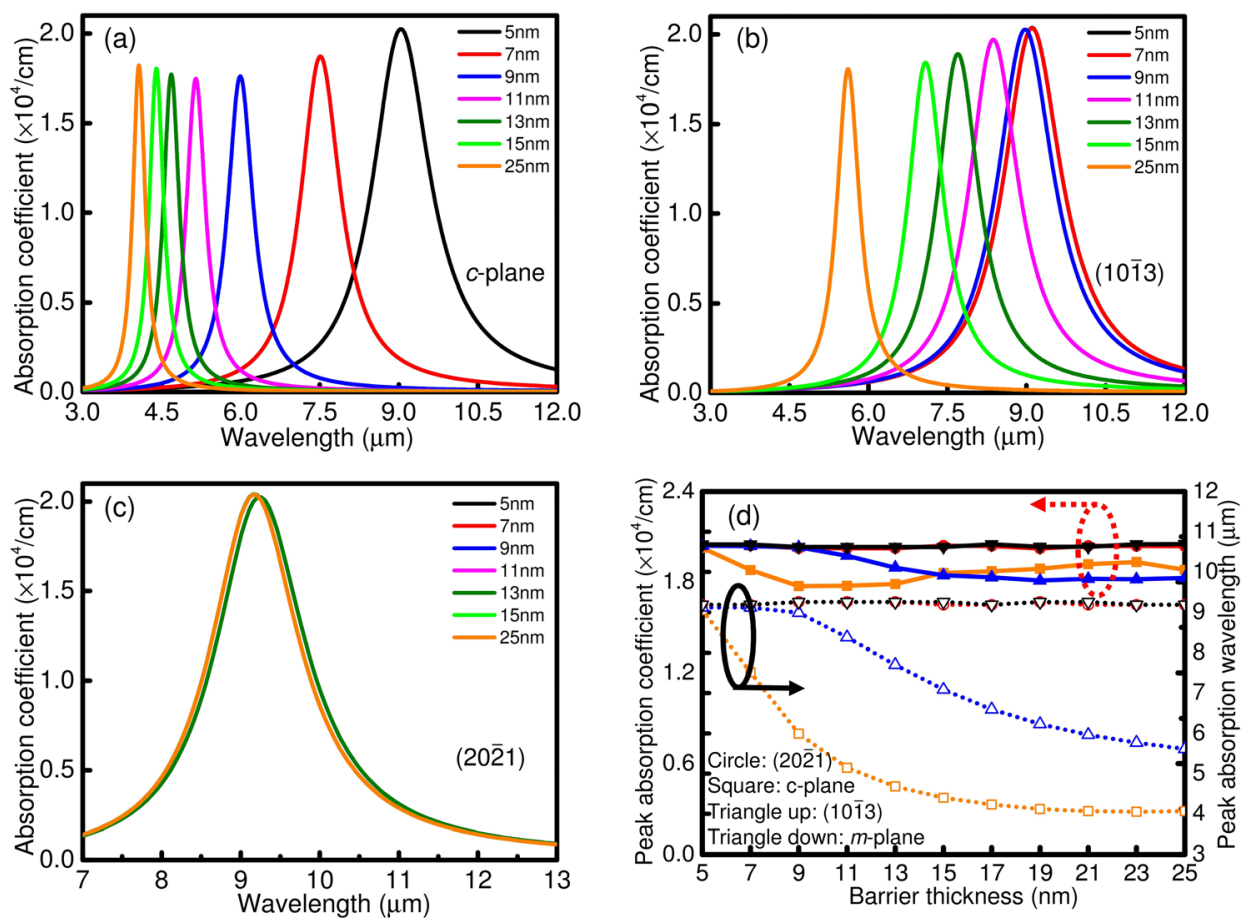

FIG. 8. Absorption spectra of (a)

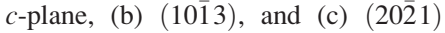
$\mathrm{Al}_{0.3} \mathrm{Ga}_{0.7} \mathrm{~N} / \mathrm{GaN}(5 \mathrm{~nm}) \mathrm{SQW}$ varying barrier thickness from $5 \mathrm{~nm}$ to $25 \mathrm{~nm}$. Most of the spectra of $(20 \overline{2} 1)$ QW with different barrier thicknesses are overlapped. (d) Peak absorption coefficient and peak absorption wavelength as a function of barrier thickness for $c$-plane, (2021), (1013), and $m$-plane $\mathrm{Al}_{0.3} \mathrm{Ga}_{0.7} \mathrm{~N} / \mathrm{GaN}$ SQW.
The influence of barrier thickness on the device performance is explained in Fig. 9, where we plot the undoped QW profile with barrier thicknesses of $9 \mathrm{~nm}, 17 \mathrm{~nm}$, and $25 \mathrm{~nm}$ for above four planes. In the case of $c$-plane and (1013) SQW, the profile gets more bent down with increasing barrier thickness. This can further push the subband wavefunctions apart, resulting in an increased ISBT frequency and decreased dipole matrix elements. However, the

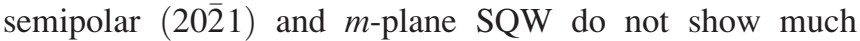
change in the QW profile when the barrier thickness is varied, which could explain the stable performance. With increasing barrier thickness, distances between polarizationinduced charges in the QWs are changed. As the result, the electric field in the QW is increased, and the electric field in the barrier is decreased. ${ }^{37,38}$ Therefore, thicker barrier of the $c$-plane and (1013) SQW produces more titling in QW and less tilting in the barrier. It is also shown that the tilting is stronger in the $c$-plane SQW than in the semipolar (1013) SQW because of stronger polarization. Due to negligible or no polarization induced charge at the interface, the QW
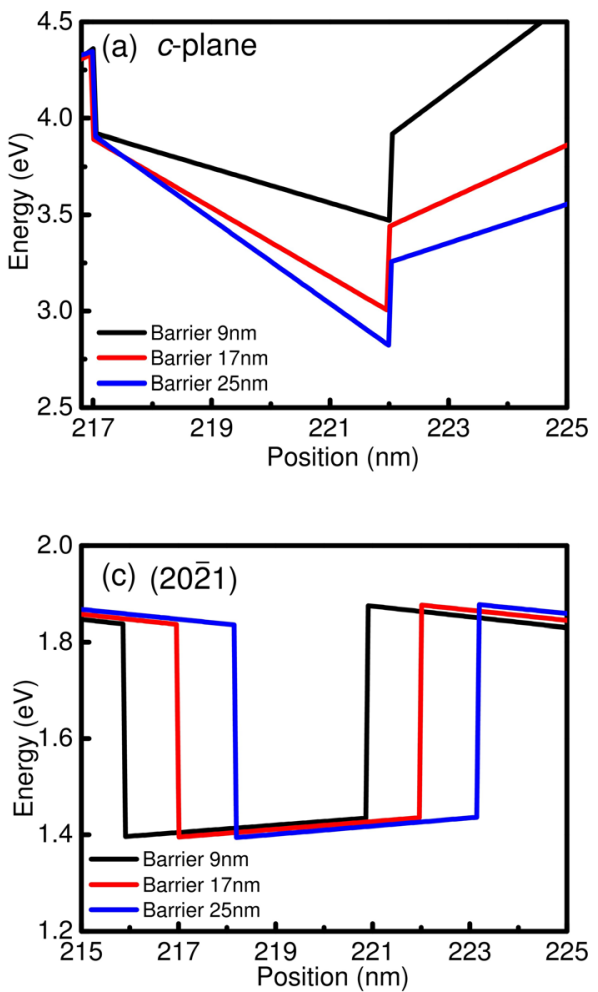
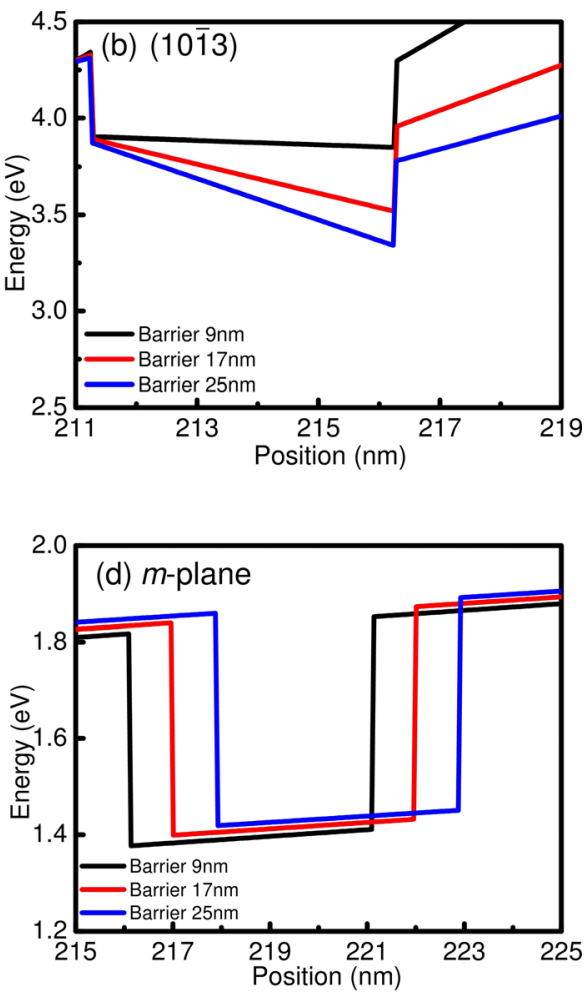

FIG. 9. Conduction band of (a) c-plane,

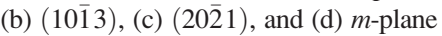
$\mathrm{Al}_{0.3} \mathrm{Ga}_{0.7} \mathrm{~N} / \mathrm{GaN}(5 \mathrm{~nm})$ undoped SQW with barrier thicknesses of $9 \mathrm{~nm}, 17 \mathrm{~nm}$, and $25 \mathrm{~nm}$. 

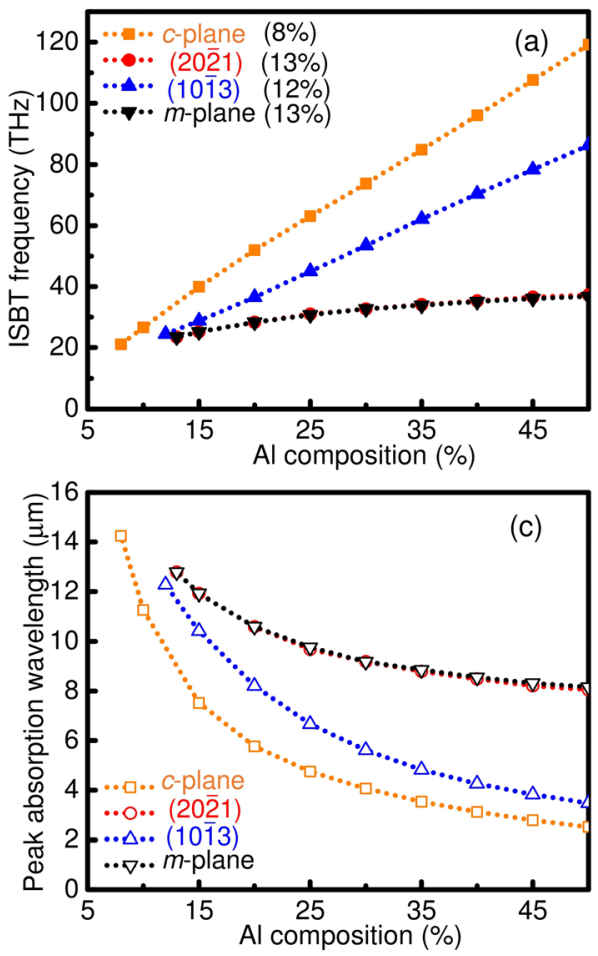
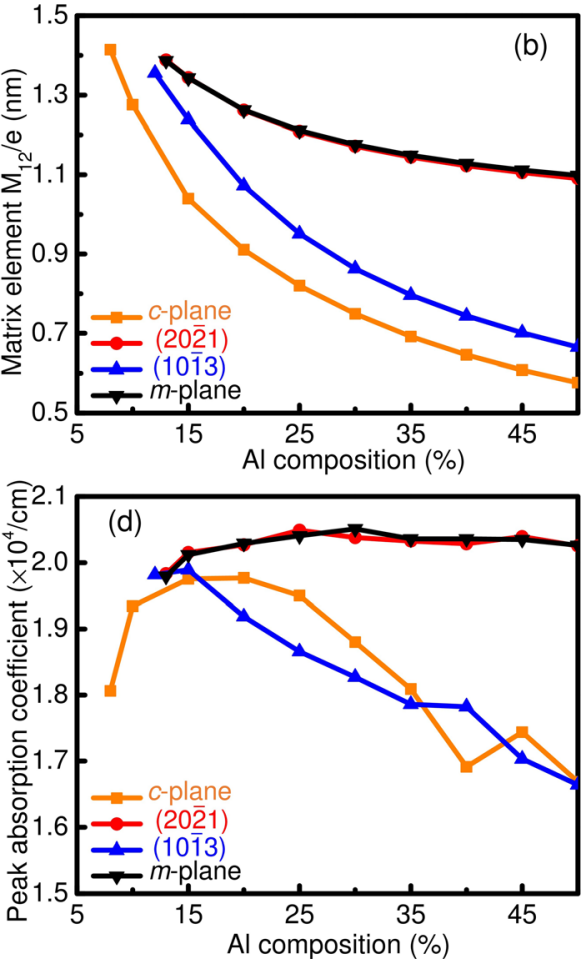

FIG. 10. (a) ISBT frequency and (b) matrix element $\mathrm{M}_{12} / e$ of $\mathrm{AlGaN}$ $(25 \mathrm{~nm}) / \mathrm{GaN}(5 \mathrm{~nm}) \mathrm{SQW}$ as a function of $\mathrm{Al}$ composition of barrier on

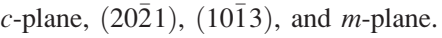
In (b), the listed composition (8\%, $13 \%, 12 \%$, and $13 \%$ ) are the minimum Al composition needed to ensure two subbands in the SQW on different planes. (c) Peak absorption wavelength and (d) peak absorption coefficient as a function of barrier $\mathrm{Al}$ composition for different SQW. profiles of semipolar $(20 \overline{2} 1)$ and $m$-plane are not influenced by barrier thickness.

\section{Barrier Al composition}

We also study the effect of barrier Al composition on the ISBT properties of the semipolar SQW. Figures 10(a) and 10(b) show the ISBT frequency and dipole matrix elements, respectively. The Al composition next to the legends are the minimum $\mathrm{Al}$ composition required to have two subbands in the QW. With increasing $\mathrm{Al}$ composition, the transition frequencies of the semipolar (1013) and $c$-plane SQW experience a dramatic increase, while those of $(20 \overline{2} 1)$ and $m$-plane SQW are mostly stable. This is because high $\mathrm{Al}$ composition will lead to strong $\Delta \mathrm{P}_{\mathrm{pz}}$ and $\Delta \mathrm{P}_{\text {tot }}$, which make the QW profile more tilted for the semipolar (1013) and $c$-plane SQW. Furthermore, the dipole matrix elements on the semipolar (1013) and $c$-plane SQW are also largely reduced by high $\mathrm{Al}$ composition in the barrier. Figures 10(c) and $10(\mathrm{~d})$ describe the peak absorption coefficients and peak absorption wavelength as a function of $\mathrm{Al}$ composition. The peak absorption wavelength is decreasing with higher $\mathrm{Al}$ composition. But the wavelength reduction is relatively smaller for the semipolar $(20 \overline{2} 1)$ SQW than the (1013) and $c$-plane SQW. And the peak absorption coefficients of $(20 \overline{2} 1)$ SQW remain constant with increasing Al composition. This indicates that at the same $\mathrm{Al}$ composition, the semipolar (202 1 ) SQW with high absorption coefficients will behave much better in long wavelength optoelectronics.

\section{CONCLUSIONS}

We study the ISBT properties of semipolar AlGaN/GaN SQW. The semipolar planes in the range of $55^{\circ}<\theta<90^{\circ}$ exhibit a unique shorter ISBT frequency and longer wavelength response. In addition, they have high absorption coefficients and absorption quantum efficiency, and has lower requirement in QW structures to absorb 50\% incident light. The effect of QW thickness, barrier thickness, and barrier Al composition on the ISBT properties of the semipolar $(20 \overline{2} 1)$ (weak polarization) and semipolar (1013) (strong polarization) SQW are studied in detail. The semipolar $(20 \overline{2} 1)$ SQW shows tunable transition frequency and absorption wavelength with high absorption coefficients when varying QW thickness. It also shows stable device performance when the barrier thickness and Al composition are changed. All these results indicate that semipolar planes with weak polarization can be used to fabricate high performance low frequency and long wavelength optoelectronic devices.

\section{ACKNOWLEDGMENTS}

This work was supported by Bisgrove Scholar program from Science Foundation Arizona.

${ }^{1}$ S. Nakamura, T. Mukai, and M. Senoh, Appl. Phys. Lett. 64, 1687 (1994).

${ }^{2}$ S. Nakamura, G. Fasol, and S. J. Pearton, The Blue Laser Diode: The Complete Story, 2nd ed. (Springer, 2000).

${ }^{3}$ Y. Zhao, J. Sonoda, I. Koslow, C. C. Pan, H. Ohta, J. S. Ha, S. P. Denbaars, and S. Nakamura, Jpn. J. Appl. Phys., Part 1 49, 070206 (2010).

${ }^{4}$ Y. Enya, Y. Yoshizumi, T. Kyono, K. Akita, M. Ueno, M. Adachi, T. Sumitomo, S. Tokuyama, T. Ikegami, K. Katayama, and T. Nakamura, Appl. Phys. Express 2, 082101 (2009).

${ }^{5}$ Y. Zhao, S. H. Oh, F. Wu, Y. Kawaguchi, S. Tanaka, K. Fujito, J. S. Speck, S. P. DenBaars, and S. Nakamura, Appl. Phys. Express 6, 062102 (2013).

${ }^{6}$ D. Hofstetter, S. Schad, H. Wu, W. Schaff, and L. Eastman, Appl. Phys. Lett. 83, 572 (2003).

${ }^{7}$ S. Sakr, E. Giraud, A. Dussaigne, M. Tchernycheva, N. Grandjean, and F. Julien, Appl. Phys. Lett. 100, 181103 (2012).

${ }^{8}$ E. Bellotti, K. Driscoll, T. Moustakas, and R. Paiella, Appl. Phys. Lett. 92, 101112 (2008).

${ }^{9}$ H. C. Liu, C. Y. Song, A. J. SpringThorpe, and J. C. Cao, Appl. Phys. Lett. 84, 4068 (2004). 
${ }^{10}$ N. Horiuchi, Nat. Photonics 4, 140 (2010).

${ }^{11}$ A. Romanov, T. Baker, S. Nakamura, and J. Speck, J. Appl. Phys. 100, 023522 (2006).

${ }^{12}$ N. Iizuka, K. Kaneko, and N. Suzuki, Appl. Phys. Lett. 81, 1803 (2002).

${ }^{13}$ C. Gmachl, H. M. Ng, and A. Y. Cho, Appl. Phys. Lett. 77, 334 (2000).

${ }^{14}$ H. Machhadani, Y. Kotsar, S. Sakr, M. Tchernycheva, R. Colombelli, J. Mangeney, E. Bellet-Amalric, E. Sargiannidou, E. Monroy, and F. H. Julien, Appl. Phys. Lett. 97, 191101 (2010).

${ }^{15}$ D. Feezell, Y. Sharma, and S. Krishna, J. Appl. Phys. 113, 133103 (2013).

${ }^{16}$ A. Pesach, E. Gross, C. Y. Huang, Y. D. Lin, A. Vardi, S. E. Schacham, and G. Bahir, Appl. Phys. Lett. 103, 022110 (2013).

${ }^{17}$ C. Edmunds, J. Shao, M. Shirazi-HD, M. J. Manfra, and O. Malis, Appl. Phys. Lett. 105, 021109 (2014).

${ }^{18}$ T. Kotani, M. Arita, and Y. Arakawa, Appl. Phys. Lett. 105, 261108 (2014).

${ }^{19}$ M. B. Mclaurin, A. Hirai, E. Young, F. Wu, and J. S. Speck, Jpn. J. Appl. Phys., Part 1 47, 5429-5431 (2008).

${ }^{20}$ F. Wu, Y. D. Lin, A. Chakraborty, H. Ohta, S. P. DenBaars, S. Nakamura, and J. S. Speck, Appl. Phys. Lett. 96, 231912 (2010).

${ }^{21}$ Y. Zhao, Q. Yan, C. Huang, S. Huang, P. Hsu, S. Tanaka, C. Pan, Y. Kawaguchi, K. Fujito, C. G. Van de Walle, J. Speck, S. DenBaars, S. Nakamura, and D. Feezell, Appl. Phys. Lett. 100, 201108 (2012).

${ }^{22}$ Y. Zhao, Q. Yan, D. Feezell, K. Fujito, C. G. Van de Walle, J. S. Speck, S. P. DenBaars, and S. Nakamura, Opt. Express 21, A53 (2013).

${ }^{23}$ Y. Zhao, S. Tanaka, Q. Yan, C. Y. Huang, R. B. Chung, C. C. Pan, K. Fujito, D. Feezell, C. G. Van de Walle, J. S. Speck, S. P. DenBaars, and S. Nakamura, Appl. Phys. Lett. 99, 051109 (2011).
${ }^{24}$ H. Fu, Z. Lu, X. Zhao, Y. Zhang, S. P. DenBaars, S. Nakamura, and Y. Zhao, J. Disp. Technol. PP(99), 1 (2016).

${ }^{25}$ Y. Zhao, S. Tanaka, C. C. Pan, K. Fujito, D. Feezell, J. S. Speck, S. P. DenBaars, and S. Nakamura, Appl. Phys. Express 4, 082104 (2011).

${ }^{26}$ L. Lahourcade, P. K. Kandaswamy, J. Renard, P. Ruterana, H. Machhadani, M. Tchernycheva, F. H. Julien, B. Gayral, and E. Monroy, Appl. Phys. Lett. 93, 111906 (2008).

${ }^{27}$ H. Machhadani, M. Beeler, S. Sakr, E. Warde, Y. Kotsar, M. Tchernycheva, M. P. Chauvat, P. Ruterana, G. Nataf, Ph. De Mierry, E. Monroy, and F. H. Julien, J. Appl. Phys. 113, 143109 (2013).

${ }^{28}$ V. F. Mymrin, K. A. Bulashevich, N. I. Podolskaya, I. A. Zhmakin, S. Yu. Karpov, and Yu. N. Makarov, Phys. Status Solidi C 2, 2928 (2005).

${ }^{29}$ S. H. Park and S. L. Chuang, Appl. Phys. Lett. 76, 1981 (2000).

${ }^{30}$ See http://www.str-soft.com/products/SiLENSe/ for more information about physical models and related publications.

${ }^{31}$ D. Ahn and S. Chuang, IEEE J. Quantum Electron. 23, 2196 (1987).

${ }^{32} \mathrm{H}$. Schneider and H. Liu, Quantum Well Infrared Photodetectors: Physics and Applications (Springer, Berlin, 2006).

${ }^{33}$ S. H. Park, J. Appl. Phys. 91, 9904 (2002).

${ }^{34}$ E. Ejder, Phys. Status Solidi A 6, 445-448 (1971).

${ }^{35}$ T. Kawashima, H. Yoshikawa, and S. Adachi, J. Appl. Phys. 82, 3528 (1997).

${ }^{36}$ C. C. Pan, S. Tanaka, F. Wu, Y. Zhao, J. S. Speck, S. Nakamura, S. P. DenBaars, and D. Feezell, Appl. Phys. Express 5, 062103 (2012).

${ }^{37}$ J. J. Wierer, Jr., D. D. Koleske, and S. R. Lee, Appl. Phys. Lett. 100, 111119 (2012).

${ }^{38}$ G. B. Lin, D. Y. Kim, G. Shan, J. Cho, E. F. Schubert, H. Shim, C. Sone, and J. K. Kim, IEEE Photonics J. 5, 1600207 (2013). 